CRYSTALLOGRAPHIC COMMUNICATIONS

ISSN 2056-9890

Received 2 September 2021

Accepted 6 September 2021

Edited by B. Therrien, University of Neuchâtel, Switzerland

Keywords: crystal structure; serine protease inhibitor; treatment of serve acute pancreatitis; anticoagulant agent.

CCDC reference: 2107852

Supporting information: this article has supporting information at journals.iucr.org/e

\section{Crystal structure of nafamostat dimesylate}

\author{
Isao Fujii*
}

STEM Education Center, Tokai University, 4-1-1 Kitakaname, Hiratuka, Kanagawa 259-1292, Japan. *Correspondence e-mail: fujii@wing.ncc.u-tokai.ac.jp

Nafamostat dimesylate \{systematic name: [amino(\{6-[(4-\{[amino(iminiumyl)methyl]amino\}phenyl)carbonyloxy]naphthalen-2-yl\})methylidene]azanium bis(methanesulfonate)\}, $\mathrm{C}_{19} \mathrm{H}_{19} \mathrm{~N}_{5} \mathrm{O}_{2}{ }^{2+} \cdot 2 \mathrm{CH}_{3} \mathrm{O}_{3} \mathrm{~S}^{-}$, is a broad-spectrum serine protease inhibitor and has been applied clinically as an anticoagulant agent during hemodialysis and for treatment of severe acute pancreatitis (SAP). Since nafamostat contains flexible moieties, it is necessary to determine the conformation to understand the structure-activity relationships. The divalent cation has a screw-like motif. The guanidinium group is approximately perpendicular to the naphthyl ring system, subtending a dihedral angle of $84.30(14)^{\circ}$. In the crystal, the nafamostat molecules form columnar structures surrounded by a hydrophilic region.

\section{Chemical context}

Nafamostat mesylate (I) is the bismethanesulfonic salt of 6amidino-2-naphthyl-4-guanidinobenzoate. It shows broadspectrum serine protease inhibition effect, and is also a reversible competitive inhibitor as camostat mesylate (II) (Tamura et al., 1977; Fujii \& Hitomi, 1981; Matsumoto et al., 1989). Although nafamostat mesylate has been applied clinically with success as an effective anticoagulant and antiinflammatory agent during hemodialysis and for treatment of severe acute pancreatitis (Takeda et al., 1989), the crystal structure has not previously been reported.<smiles>NC(=[NH2+])Nc1ccc(C(=O)Oc2ccc3cc(C(N)=[NH2+])ccc3c2)cc1</smiles><smiles>CS(=O)(=O)[Hg]1CCCCC1</smiles>

Nafamostat mesylate (I)<smiles>CN(C)C(=O)COC(=O)Cc1ccc(OC(=O)c2ccc(NC(N)=[NH2+])cc2)cc1</smiles>

Camostat mesylate (II)

In addition, nafamostat has attracted attention as an inhibitor for the activity of transmembrane protease serine 2 (TMPRSS2), a host cell serine protease that mediates viral cell incursion for influenza virus and coronavirus, thereby inhibiting viral infection and replication (Yamamoto et al., 2016, 2020; Hoffmann et al., 2020). Since nafamostat contains flex- 
Table 1

Selected bond lengths $(\AA)$.

\begin{tabular}{llll}
\hline $\mathrm{C} 1-\mathrm{O} 11$ & $1.391(3)$ & $\mathrm{C} 30-\mathrm{S} 27$ & $1.764(3)$ \\
$\mathrm{C} 12-\mathrm{O} 13$ & $1.204(3)$ & $\mathrm{C} 35-\mathrm{S} 32$ & $1.762(3)$ \\
$\mathrm{C} 12-\mathrm{O} 11$ & $1.377(3)$ & $\mathrm{O} 28-\mathrm{S} 27$ & $1.4515(19)$ \\
$\mathrm{C} 14-\mathrm{N} 22$ & $1.311(3)$ & $\mathrm{O} 29-\mathrm{S} 27$ & $1.4570(19)$ \\
$\mathrm{C} 14-\mathrm{N} 15$ & $1.319(3)$ & $\mathrm{O} 31-\mathrm{S} 27$ & $1.4700(18)$ \\
$\mathrm{C} 19-\mathrm{N} 23$ & $1.417(3)$ & $\mathrm{O} 33-\mathrm{S} 32$ & $1.4545(18)$ \\
$\mathrm{C} 24-\mathrm{N} 26$ & $1.325(3)$ & $\mathrm{O} 34-\mathrm{S} 32$ & $1.4553(19)$ \\
$\mathrm{C} 24-\mathrm{N} 25$ & $1.302(4)$ & $\mathrm{O} 36-\mathrm{S} 32$ & $1.448(2)$ \\
$\mathrm{C} 24-\mathrm{N} 23$ & $1.357(3)$ & &
\end{tabular}

ible moieties, it is necessary to determine the conformation to understand the structure-activity relationships. The crystal structure of nafamostat mesylate (I) is reported herein. From the crystallographic study, the phenylguanidine groups in nafamostat and camostat are essentially similar except for the direction of residual groups.

\section{Structural commentary}

The nafamostat moiety in the title compound (Fig. 1) shows a divalent cation with a screw-like motif, which consists of four planar parts: the amidino group, the naphthyl group (rings $A$ and $B$ ), phenyl ring $C$ and the guanidinium group (shown in Fig.1). The dihedral angles between the amidino and naphthyl groups, the naphthyl group and ring $C$, and ring $C$ and guanidinium group are $11.35(13), 44.66(10)$ and $51.11(15)^{\circ}$, respectively. The guanidinium group is approximately perpendicular to the naphthyl group, subtending a dihedral angle of $84.30(14)^{\circ}$.

The C14-N15 and C14-N22 bond distances [1.319 (3) and 1.311 (3) $\AA$, respectively] indicate a resonance structure in the protonated amidinium group (Table 1). On the other hand, the bond distances $\mathrm{C} 24-\mathrm{N} 23=1.357$ (3), C24-N25 = 1.302 (4) and $\mathrm{C} 24-\mathrm{N} 26=1.325$ (3) $\AA$ indicate a localized electron on the $\mathrm{C} 24-\mathrm{N} 25$ bond in the protonated guanidinium group.

The overlay of nafamostat (green) and camostat (red) is presented in Fig. 2, in which the r.m.s. deviation is $0.027 \AA$ for phenylguanidinium groups. The partial structures are essentially similar, except for the direction of residual groups. Very

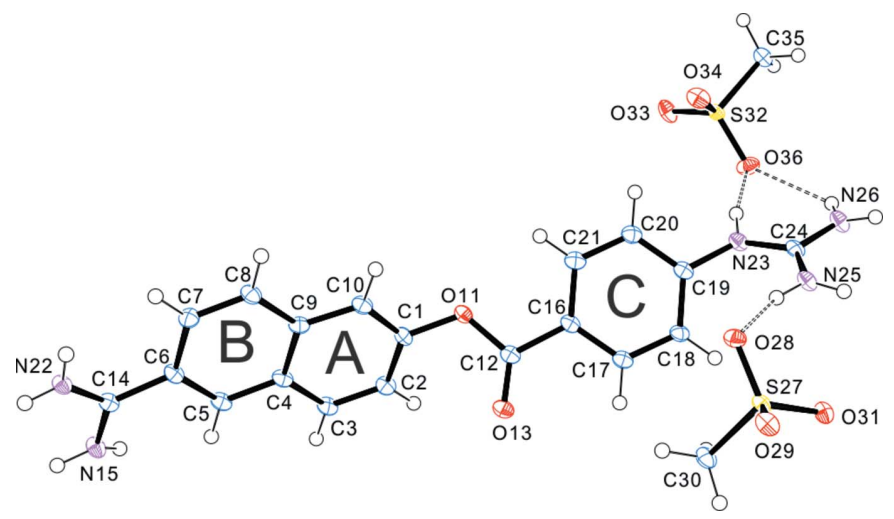

Figure 1

The title compound nafamostat mesylate (I) showing the atom and ring labelling. Displacement ellipsoids are drawn at the $50 \%$ probability level.

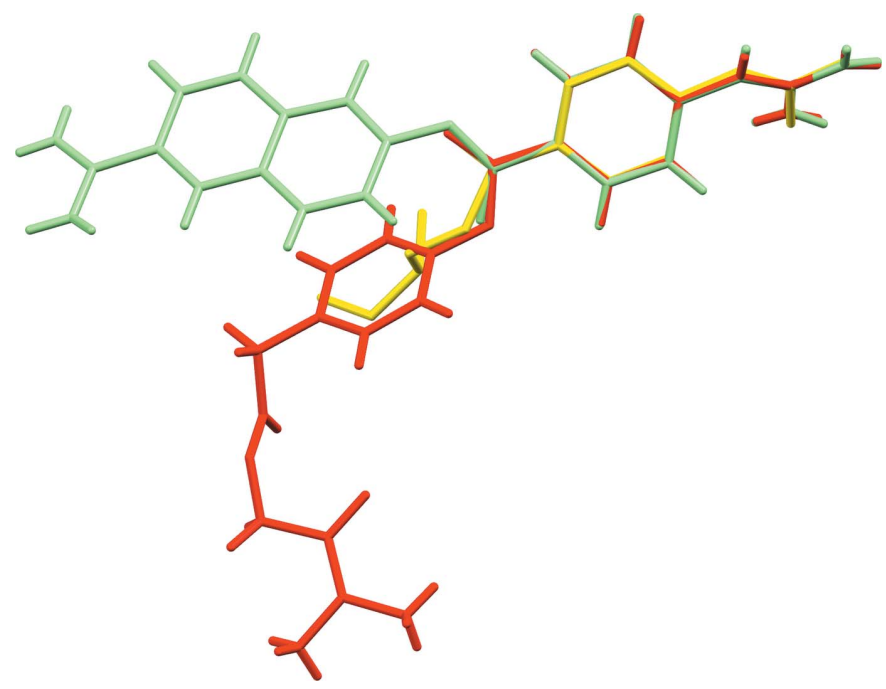

Figure 2

Overlay of the crystal structures of nafamostat moiety (green), camostat moiety (red) and covalently binding partial structure (yellow) of mature nafamostat with Ser441 in the active site from pdb7MEQ (Fraser et al., 2021), using Mercury (Macrae et al., 2020).

recently, the crystal structure of human TMPRSS2 in a covalent complex with nafamostat has been solved (Fraser et al., 2021). The nafamostat in the complex is hydrolysed, and results in phenylguanidino acylation of Ser441 (yellow) in the active site. It was considered that the nafamostat moiety may be easily nucleophilic-attacked, approaching from the O13 atom side without steric hindrance.

\section{Supramolecular features}

In the crystal, the naphthyl groups of nafamostat form hydrophobic columnar structures, shown in Fig. 3. The naph-

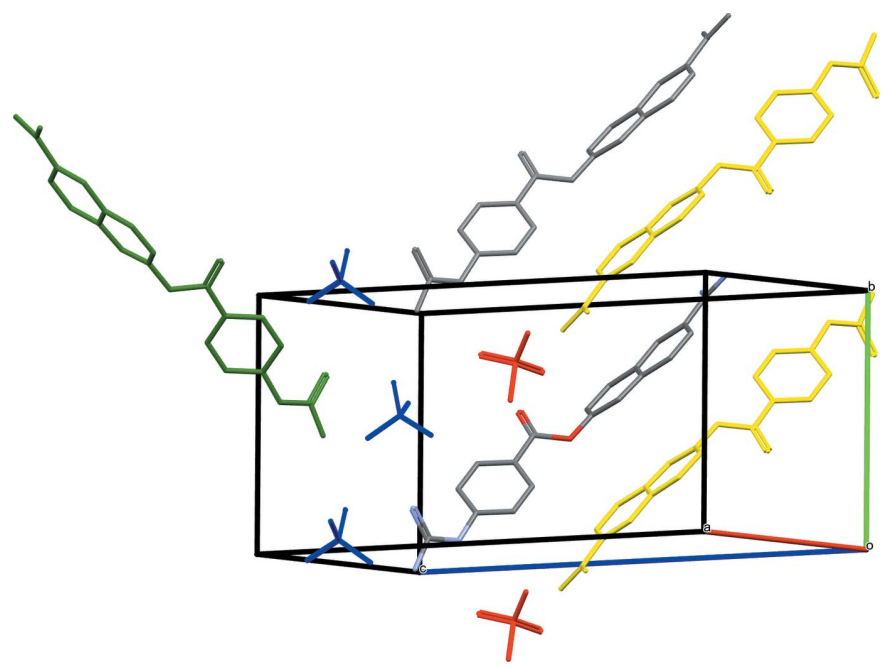

Figure 3

Part of the crystal structure of nafamostat mesylate (I). The naphthyl groups related by the inversion center (yellow) and equivalent (grey) are stacking along the $b$-axis direction, forming a columnar structure. The methanesulfonate groups containing the S27 and S32 atoms are represented in blue and red, respectively. $\mathrm{H}$ atoms have been omitted for clarity. 
Table 2

Hydrogen-bond geometry $\left(\AA,^{\circ}\right)$.

$\mathrm{Cg}(C)$ is the center of gravity of phenyl ring $C$.

\begin{tabular}{lllll}
\hline$D-\mathrm{H} \cdots A$ & $D-\mathrm{H}$ & $\mathrm{H} \cdots A$ & $D \cdots A$ & $D-\mathrm{H} \cdots A$ \\
\hline $\mathrm{C} 18-\mathrm{H} 18 \cdots \mathrm{O} 13^{\mathrm{i}}$ & 0.95 & 2.64 & $3.419(3)$ & 140 \\
$\mathrm{C} 30-\mathrm{H} 30 A \cdots \mathrm{O} 36^{\mathrm{ii}}$ & 0.98 & 2.36 & $3.314(3)$ & 165 \\
$\mathrm{~N} 15-\mathrm{H} 15 A \cdots \mathrm{O} 33^{\text {iii }}$ & $0.93(4)$ & $1.97(4)$ & $2.854(3)$ & $159(3)$ \\
$\mathrm{N} 15-\mathrm{H} 15 A \cdots \mathrm{O} 34^{\text {iii }}$ & $0.93(4)$ & $2.63(4)$ & $3.327(3)$ & $132(3)$ \\
$\mathrm{N} 15-\mathrm{H} 15 A \cdots \mathrm{S} 32^{\text {iii }}$ & $0.93(4)$ & $2.75(4)$ & $3.612(2)$ & $154(3)$ \\
$\mathrm{N} 15-\mathrm{H} 15 B \cdots \mathrm{O} 34^{\mathrm{iv}}$ & $0.85(4)$ & $2.00(4)$ & $2.830(3)$ & $164(4)$ \\
$\mathrm{N} 22-\mathrm{H} 22 A \cdots \mathrm{O} 31^{\mathrm{v}}$ & $0.83(3)$ & $2.12(3)$ & $2.928(3)$ & $162(3)$ \\
$\mathrm{N} 22-\mathrm{H} 22 B \cdots \mathrm{O} 33^{\mathrm{iii}}$ & $0.83(3)$ & $2.31(3)$ & $3.018(3)$ & $144(3)$ \\
$\mathrm{N} 23-\mathrm{H} 23 \cdots \mathrm{O} 36$ & $0.99(4)$ & $1.88(5)$ & $2.836(3)$ & $163(4)$ \\
$\mathrm{N} 23-\mathrm{H} 23 \cdots \mathrm{S} 32$ & $0.99(4)$ & $2.72(4)$ & $3.683(2)$ & $166(3)$ \\
$\mathrm{N} 25-\mathrm{H} 25 A \cdots \mathrm{O} 28$ & $0.87(4)$ & $2.00(4)$ & $2.827(3)$ & $159(3)$ \\
$\mathrm{N} 25-\mathrm{H} 25 A \cdots \mathrm{S} 27$ & $0.87(4)$ & $2.86(4)$ & $3.558(2)$ & $139(3)$ \\
$\mathrm{N} 25-\mathrm{H} 25 B \cdots \mathrm{O} 29^{\mathrm{vi}}$ & $0.83(3)$ & $2.12(3)$ & $2.931(3)$ & $167(3)$ \\
$\mathrm{N} 26-\mathrm{H} 26 A \cdots \mathrm{O} 31^{\mathrm{vi}}$ & $0.85(4)$ & $2.10(4)$ & $2.916(3)$ & $163(4)$ \\
$\mathrm{N} 26-\mathrm{H} 26 A \cdots \mathrm{S} 27^{\mathrm{vi}}$ & $0.85(4)$ & $3.01(4)$ & $3.799(2)$ & $156(3)$ \\
$\mathrm{N} 26-\mathrm{H} 26 B \cdots \mathrm{O} 29^{\text {vii }}$ & $0.89(4)$ & $2.46(4)$ & $2.925(3)$ & $113(3)$ \\
$\mathrm{N} 26-\mathrm{H} 26 B \cdots \mathrm{O} 36$ & $0.89(4)$ & $2.45(4)$ & $3.174(3)$ & $139(3)$ \\
$\mathrm{C} 30-\mathrm{H} 30 B \cdots C g(\mathrm{C})^{\text {vii }}$ & 0.98 & 2.96 & $3.405(3)$ & 109
\end{tabular}

Symmetry codes: (i) $-x+1, y-\frac{1}{2},-z+\frac{3}{2}$; (ii) $x, y+1, z$; (iii) $-x,-y+1,-z+1$; (iv) $x-1, y+1, z$; (v) $x-1,-y+\frac{3}{2}, z-\frac{1}{2}$; (vi) $-x+2, y-\frac{1}{2},-z+\frac{3}{2}$; (vii) $x, y-1, z$.

thyl groups correlated with the inversion center (yellow) are stacking along the $b$-axis direction, in which the perpendicular distances of the centroid of the naphthyl ring system and those at $(-x, 1-y, 1-z)$ and $(-x, 2-y, 1-z)$ are $3.4208(8)$ and 3.5134 (8) A, respectively.

The columnar structures are surrounded by a hydrophilic region consisting of the methanesulfonate ions and the guanidinium, imidamidium and ester groups. The two independent methanesulfonate ions play different roles. The columnar structure intercalates the methanesulfonate group (blue) containing the S27 atom, and is linked to three neighbouring guanidinium groups and one diamine group. Hydrogen bonds [N25-H25A $\cdots \mathrm{O} 28=2.827$ (3) and N26$\mathrm{H} 26 B \cdots \mathrm{O} 29^{\mathrm{vii}}=2.925$ (3) $\AA$; Table 2] link the molecules, forming an infinite $C_{2}^{2}(8)$ chain, with other hydrogen bonds $\left[\mathrm{N} 25-\mathrm{H} 25 B \cdots \mathrm{O} 29^{\mathrm{vi}}=2.931(3)\right.$ and $\mathrm{N} 26-\mathrm{H} 26 A \cdots \mathrm{O} 31^{\mathrm{vi}}=$ 2.916 (3) $\AA$ ] forming an $R_{2}^{2}(8)$ ring.

The columnar structures are also consolidated by the other methanesulfonate ion (red) containing the $\mathrm{S} 32$ atom, which is linked by two opposing amidino groups [N15-H15A ...O33 $3^{\text {iii }}$ $=2.854$ (3) and $\mathrm{N} 15-\mathrm{H} 15 B \cdots \mathrm{O} 34^{\mathrm{iv}}=2.830$ (3) $\AA$ ], related by the inversion center, into an $R_{4}^{4}(12)$ ring. A weak $\mathrm{C}-\mathrm{H} \cdots \pi$ interaction is also observed (Table 2).

\section{Database survey}

The crystal structures of serine protease inhibitors have been reported for benzamidine (TEKTUY: Barker et al., 1996), benzamidine $\mathrm{HCl}$ (DOHHAJ: Thailambal et al., 1986) and camostat mesylate (JAMREU: Matsumoto et al., 1989). Moreover, a search of the Cambridge Structural Database (CSD version 5.42, last updated May 2021; Groom et al., 2016) yielded another comparable structure, 4-guanidiniobenzoic acid $\mathrm{HCl}$ dihydrate (NIQCEW: Light et al., 2007). Another database search (PDB; Berman et al., 2000) yielded the crystal
Table 3

Experimental details.

\begin{tabular}{|c|c|}
\hline \multicolumn{2}{|l|}{ Crystal data } \\
\hline Chemical formula & $\mathrm{C}_{19} \mathrm{H}_{19} \mathrm{~N}_{5} \mathrm{O}_{2}{ }^{2+} \cdot 2 \mathrm{CH}_{3} \mathrm{O}_{3} \mathrm{~S}^{-}$ \\
\hline$M_{\mathrm{r}}$ & 539.58 \\
\hline Crystal system, space group & Monoclinic, $P 2_{1} / c$ \\
\hline Temperature $(\mathrm{K})$ & 95 \\
\hline$a, b, c(\AA)$ & 11.0631 (1), 9.7215 (1), 21.9271 (3) \\
\hline$\beta\left(^{\circ}\right)$ & $96.746(1)$ \\
\hline$V\left(\AA^{3}\right)$ & $2341.93(5)$ \\
\hline$Z$ & 4 \\
\hline Radiation type & $\mathrm{Cu} K \alpha$ \\
\hline$\mu\left(\mathrm{mm}^{-1}\right)$ & 2.59 \\
\hline Crystal size $(\mathrm{mm})$ & $0.4 \times 0.3 \times 0.3$ \\
\hline \multicolumn{2}{|l|}{ Data collection } \\
\hline Diffractometer & A Rigaku XtaLAB P200 \\
\hline Absorption correction & $\begin{array}{l}\text { Multi-scan (CrysAlis PRO; Rigaku, } \\
\text { 2015) }\end{array}$ \\
\hline$T_{\min }, T_{\max }$ & $0.46,1$ \\
\hline $\begin{array}{l}\text { No. of measured, independent and } \\
\text { observed }[I>2 \sigma(I)] \text { reflections }\end{array}$ & $4675,4675,4457$ \\
\hline$R_{\text {int }}$ & 0.058 \\
\hline$(\sin \theta / \lambda)_{\max }\left(\AA^{-1}\right)$ & 0.623 \\
\hline \multicolumn{2}{|l|}{ Refinement } \\
\hline$R\left[F^{2}>2 \sigma\left(F^{2}\right)\right], w R\left(F^{2}\right), S$ & $0.055,0.126,1.07$ \\
\hline No. of reflections & 4675 \\
\hline No. of parameters & 363 \\
\hline $\mathrm{H}$-atom treatment & $\begin{array}{l}\mathrm{H} \text { atoms treated by a mixture of } \\
\text { independent and constrained } \\
\text { refinement }\end{array}$ \\
\hline$\Delta \rho_{\max }, \Delta \rho_{\min }\left(\mathrm{e} \AA^{-3}\right)$ & $0.64,-0.52$ \\
\hline
\end{tabular}

Computer programs: CrysAlis PRO (Rigaku, 2015), SORTAV (Blessing, 1995), SHELXT (Sheldrick, 2015a), SHELXL (Sheldrick, 2015b), ORTEP-3 for Windows and WinGX (Farrugia, 2012), Mercury (Macrae et al., 2020), PLATON (Spek, 2020) and publCIF (Westrip, 2010).

structure of human TMPRSS2 in a covalent complex with nafamostat (PDB7MEQ: Fraser et al., 2021).

\section{Synthesis and crystallization}

Nafamostat mesylate (CAS No. 82956-11-4) was purchased from Tokyo Chemical Industry Co. Ltd (TCI). A small portion (ca $10 \mathrm{mg}$ ) was dissolved in a small volume of hot water ( $c a$ $100 \mu \mathrm{L}$ ), and acetone ( $c a 900 \mu \mathrm{L}$ ) was added slowly until it became cloudy white. On slow cooling to ambient temperature, colourless octahedral crystals suitable for X-ray measurements were obtained.

\section{Refinement}

Crystal data, data collection and structure refinement details at a low temperature $(95 \mathrm{~K})$ are summarized in Table 3 . All the $\mathrm{H}$ atoms were located in difference-Fourier maps. In the $\mathrm{NH}$ or $\mathrm{NH}_{2}$ groups, $\mathrm{H}$ atoms were freely refined. The C-bound $\mathrm{H}$ atoms were included in calculated positions and treated as riding atoms: $\mathrm{C}-\mathrm{H}=0.95-0.98 \AA$ with $U_{\text {iso }}(\mathrm{H})=1.2-$ $1.5 U_{\text {eq }}(\mathrm{C})$.

\section{Acknowledgements}

The author thanks Tokai University for a research grant, which partially supported this work. 


\section{References}

Barker, J., Phillips, P. R., Wallbridge, M. G. H. \& Powell, H. R. (1996). Acta Cryst. C52, 2617-2619.

Berman, H. M., Westbrook, J., Feng, Z., Gilliland, G., Bhat, T. N., Weissig, H., Shindyalov, I. N. \& Bourne, P. E. (2000). Nucleic Acids Res. 28, 235-242.

Blessing, R. H. (1995). Acta Cryst. A51, 33-38.

Farrugia, L. J. (2012). J. Appl. Cryst. 45, 849-854.

Fraser, B., Beldar, S., Hutchinson, A., Li, Y., Seitova, A., Edwards, A. M., Benard, F., Arrowsmith, C. H. \& Halabelian, L. (2021). doi: $10.2210 / \mathrm{pdb} 7 \mathrm{MEQ} / \mathrm{pdb}$.

Fujii, S. \& Hitomi, Y. (1981). Biochim. Biophys. Acta Enzymology, 661, 342-345.

Groom, C. R., Bruno, I. J., Lightfoot, M. P. \& Ward, S. C. (2016). Acta Cryst. B72, 171-179.

Hoffmann, M., Schroeder, S., Kleine-Weber, H., Müller, M. A., Drosten, C. \& Pöhlmann, S. (2020). Antimicrob. Agents Chemother. 64, e00754-20.

Light, M. E., Martinez, J. C. \& Gale, P. A. (2007). CSD Communication (refcode NIQCEW). CCDC, Cambridge, England. https://doi.org/10.5517/ccpw2h3
Macrae, C. F., Sovago, I., Cottrell, S. J., Galek, P. T. A., McCabe, P., Pidcock, E., Platings, M., Shields, G. P., Stevens, J. S., Towler, M. \& Wood, P. A. (2020). J. Appl. Cryst. 53, 226-235.

Matsumoto, O., Taga, T. \& Machida, K. (1989). Acta Cryst. C45, $913-$ 915.

Rigaku (2015). CrysAlis PRO. Rigaku, The Woodlands, Texas, USA. Sheldrick, G. M. (2015a). Acta Cryst. A71, 3-8.

Sheldrick, G. M. (2015b). Acta Cryst. C71, 3-8.

Spek, A. L. (2020). Acta Cryst. E76, 1-11.

Takeda, K., Kakugawa, Y., Kobari, M. \& Matsuno, S. (1989). Gastroenterol. Jpn. 24, 340.

Tamura, Y., Hirado, M., Okamura, K., Minato, Y. \& Fujii, S. (1977). Biochim. Biophys. Acta Enzymology, 484, 417-422.

Thailambal, V. G., Pattabhi, V. \& Guru Row, T. N. (1986). Acta Cryst. C42, 587-589.

Westrip, S. P. (2010). J. Appl. Cryst. 43, 920-925.

Yamamoto, M., Kiso, M., Sakai-Tagawa, Y., Iwatsuki-Horimoto, K., Imai, M., Takeda, M., Kinoshita, N., Ohmagari, N., Gohda, J., Semba, K., Matsuda, Z., Kawaguchi, Y., Kawaoka, Y. \& Inoue, J. (2020). Viruses, 12, 629-638.

Yamamoto, M., Matsuyama, S., Li, X., Takeda, M., Kawaguchi, Y., Inoue, J. \& Matsuda, Z. (2016). Antimicrob. Agents Chemother. 60, 6532-6539. 


\section{supporting information}

Acta Cryst. (2021). E77, 999-1002 [https://doi.org/10.1107/S2056989021009245]

\section{Crystal structure of nafamostat dimesylate}

\section{Isao Fujii}

\section{Computing details}

Data collection: CrysAlis PRO (Rigaku, 2015); cell refinement: CrysAlis PRO (Rigaku, 2015); data reduction: SORTAV (Blessing, 1995); program(s) used to solve structure: SHELXT (Sheldrick, 2015a); program(s) used to refine structure: SHELXL (Sheldrick, 2015b); molecular graphics: ORTEP-3 for Windows (Farrugia, 2012), Mercury (Macrae et al., 2020); software used to prepare material for publication: PLATON (Spek, 2020), WinGX (Farrugia, 2012) and publCIF (Westrip, 2010).

[Amino(\{6-[(4-\{[amino(iminiumyl)methyl]amino\}phenyl)carbonyloxy]naphthalen-2-yl\})methylidene]azanium bis(methanesulfonate)

Crystal data

$\mathrm{C}_{19} \mathrm{H}_{19} \mathrm{~N}_{5} \mathrm{O}_{2}{ }^{2+} \cdot 2 \mathrm{CH}_{3} \mathrm{O}_{3} \mathrm{~S}^{-}$

$M_{r}=539.58$

Monoclinic, $P 2_{1} / c$

Hall symbol: -P 2ybc

$a=11.0631$ (1) $\AA$

$b=9.7215(1) \AA$

$c=21.9271(3) \AA$

$\beta=96.746(1)^{\circ}$

$V=2341.93(5) \AA^{3}$

$Z=4$

\section{Data collection}

A Rigaku XtaLAB P200 diffractometer

Radiation source: fine-focus sealed X-ray tube Graphite monochromator $\varphi$ or $\omega$ oscillation scans

Absorption correction: multi-scan

(CrysAlis PRO; Rigaku, 2015)

$T_{\min }=0.46, T_{\max }=1$

Refinement

Refinement on $F^{2}$

Least-squares matrix: full

$R\left[F^{2}>2 \sigma\left(F^{2}\right)\right]=0.055$

$w R\left(F^{2}\right)=0.126$

$S=1.07$

4675 reflections

363 parameters

0 restraints
$F(000)=1128$

$D_{\mathrm{x}}=1.53 \mathrm{Mg} \mathrm{m}^{-3}$

$\mathrm{Cu} K \alpha$ radiation, $\lambda=1.54184 \AA$

Cell parameters from 17552 reflections

$\theta=5.0-73.5^{\circ}$

$\mu=2.59 \mathrm{~mm}^{-1}$

$T=95 \mathrm{~K}$

Octahedron, clear light colourless

$0.4 \times 0.3 \times 0.3 \mathrm{~mm}$

4675 measured reflections

4675 independent reflections

4457 reflections with $I>2 \sigma(I)$

$R_{\text {int }}=0.058$

$\theta_{\max }=73.8^{\circ}, \theta_{\min }=4.0^{\circ}$

$h=-13 \rightarrow 13$

$k=0 \rightarrow 11$

$l=0 \rightarrow 27$

0 constraints

Primary atom site location: dual

Secondary atom site location: dual

Hydrogen site location: mixed

$\mathrm{H}$ atoms treated by a mixture of independent

and constrained refinement

$w=1 /\left[\sigma^{2}\left(F_{\mathrm{o}}^{2}\right)+(0.0324 P)^{2}+4.9921 P\right]$

where $P=\left(F_{\mathrm{o}}^{2}+2 F_{\mathrm{c}}^{2}\right) / 3$ 
$(\Delta / \sigma)_{\max }=0.001$

$\Delta \rho_{\min }=-0.52$ e $\AA^{-3}$

$\Delta \rho_{\max }=0.64 \mathrm{e} \AA^{-3}$

Special details

Geometry. All esds (except the esd in the dihedral angle between two 1.s. planes) are estimated using the full covariance matrix. The cell esds are taken into account individually in the estimation of esds in distances, angles and torsion angles; correlations between esds in cell parameters are only used when they are defined by crystal symmetry. An approximate (isotropic) treatment of cell esds is used for estimating esds involving l.s. planes.

Fractional atomic coordinates and isotropic or equivalent isotropic displacement parameters $\left(\AA^{2}\right)$

\begin{tabular}{|c|c|c|c|c|}
\hline & $x$ & $y$ & $z$ & $U_{\text {iso }} * / U_{\text {eq }}$ \\
\hline $\mathrm{C} 1$ & $0.1422(2)$ & $0.5583(3)$ & $0.56523(13)$ & $0.0225(5)$ \\
\hline $\mathrm{C} 2$ & $0.0580(2)$ & $0.5892(3)$ & $0.60620(13)$ & $0.0237(5)$ \\
\hline $\mathrm{H} 2$ & 0.061778 & 0.544066 & 0.644799 & $0.028^{*}$ \\
\hline $\mathrm{C} 3$ & $-0.0304(2)$ & $0.6865(3)$ & $0.58945(12)$ & $0.0234(5)$ \\
\hline $\mathrm{H} 3$ & -0.086637 & 0.710328 & 0.617287 & $0.028^{*}$ \\
\hline $\mathrm{C} 4$ & $-0.0384(2)$ & $0.7505(3)$ & $0.53201(12)$ & $0.0225(5)$ \\
\hline $\mathrm{C} 5$ & $-0.1241(2)$ & $0.8550(3)$ & $0.51578(12)$ & $0.0229(5)$ \\
\hline H5 & -0.177017 & 0.883065 & 0.544597 & $0.027^{*}$ \\
\hline C6 & $-0.1333(2)$ & $0.9184(3)$ & $0.45831(13)$ & $0.0229(5)$ \\
\hline $\mathrm{C} 7$ & $-0.0545(2)$ & $0.8762(3)$ & $0.41562(12)$ & $0.0237(5)$ \\
\hline H7 & -0.060246 & 0.917718 & 0.376162 & $0.028^{*}$ \\
\hline $\mathrm{C} 8$ & $0.0302(2)$ & $0.7756(3)$ & $0.43105(12)$ & $0.0228(5)$ \\
\hline $\mathrm{H} 8$ & 0.081746 & 0.747936 & 0.401556 & $0.027^{*}$ \\
\hline C9 & $0.0437(2)$ & $0.7116(3)$ & $0.48926(12)$ & $0.0221(5)$ \\
\hline $\mathrm{C} 10$ & $0.1352(2)$ & $0.6151(3)$ & $0.50758(12)$ & $0.0224(5)$ \\
\hline $\mathrm{H} 10$ & 0.191808 & 0.589251 & 0.480271 & $0.027^{*}$ \\
\hline $\mathrm{C} 12$ & $0.3080(2)$ & $0.4764(3)$ & $0.63457(12)$ & $0.0205(5)$ \\
\hline $\mathrm{C} 14$ & $-0.2214(2)$ & $1.0326(3)$ & $0.44424(12)$ & $0.0203(5)$ \\
\hline $\mathrm{C} 16$ & $0.4005(2)$ & $0.3658(3)$ & $0.64313(12)$ & $0.0206(5)$ \\
\hline $\mathrm{C} 17$ & $0.4753(2)$ & $0.3600(3)$ & $0.69882(12)$ & $0.0223(5)$ \\
\hline H17 & 0.466534 & 0.426978 & 0.729554 & $0.027^{*}$ \\
\hline $\mathrm{C} 18$ & $0.5632(2)$ & $0.2563(3)$ & $0.70998(12)$ & $0.0222(5)$ \\
\hline $\mathrm{H} 18$ & 0.61345 & 0.252049 & 0.748167 & $0.027^{*}$ \\
\hline C19 & $0.5758(2)$ & $0.1599(3)$ & $0.66434(12)$ & $0.0217(5)$ \\
\hline $\mathrm{C} 20$ & $0.5019(2)$ & $0.1658(3)$ & $0.60820(12)$ & $0.0237(5)$ \\
\hline $\mathrm{H} 20$ & 0.51147 & 0.100093 & 0.577062 & $0.028 *$ \\
\hline $\mathrm{C} 21$ & $0.4145(2)$ & $0.2678(3)$ & $0.59814(12)$ & $0.0229(5)$ \\
\hline $\mathrm{H} 21$ & 0.363537 & 0.271047 & 0.560152 & $0.027^{*}$ \\
\hline $\mathrm{C} 24$ & $0.7756(2)$ & $0.0499(3)$ & $0.69864(12)$ & $0.0212(5)$ \\
\hline $\mathrm{C} 30$ & $0.7107(2)$ & $0.5832(3)$ & $0.76962(13)$ & $0.0245(6)$ \\
\hline $\mathrm{H} 30 \mathrm{~A}$ & 0.661242 & 0.637008 & 0.738122 & $0.037 *$ \\
\hline H30B & 0.734263 & 0.641357 & 0.805571 & $0.037^{*}$ \\
\hline $\mathrm{H} 30 \mathrm{C}$ & 0.663287 & 0.504665 & 0.781635 & $0.037^{*}$ \\
\hline $\mathrm{C} 35$ & $0.6293(2)$ & $-0.4167(3)$ & $0.57039(13)$ & $0.0262(6)$ \\
\hline $\mathrm{H} 35 \mathrm{~A}$ & 0.717501 & -0.403331 & 0.578279 & $0.039 *$ \\
\hline H35B & 0.607172 & -0.441466 & 0.527188 & $0.039^{*}$ \\
\hline $\mathrm{H} 35 \mathrm{C}$ & 0.604676 & -0.490755 & 0.596665 & $0.039 *$ \\
\hline
\end{tabular}




$\begin{array}{lllll}\mathrm{N} 15 & -0.3033(2) & 1.0565(3) & 0.48204(11) & 0.0238(5) \\ \mathrm{N} 22 & -0.2175(2) & 1.1128(2) & 0.39642(11) & 0.0214(5) \\ \mathrm{N} 23 & 0.65733(19) & 0.0475(2) & 0.67377(11) & 0.0242(5) \\ \mathrm{N} 25 & 0.8365(2) & 0.1631(2) & 0.71094(12) & 0.0242(5) \\ \mathrm{N} 26 & 0.8283(2) & -0.0718(2) & 0.70825(12) & 0.0252(5) \\ \text { O11 } & 0.23387(15) & 0.46189(19) & 0.57993(8) & 0.0217(4) \\ \text { O13 } & 0.29656(16) & 0.5684(2) & 0.67019(9) & 0.0255(4) \\ \text { O28 } & 0.80155(16) & 0.44602(19) & 0.68474(8) & 0.0234(4) \\ \text { O29 } & 0.91327(16) & 0.64512(19) & 0.72854(9) & 0.0252(4) \\ \text { O31 } & 0.90733(15) & 0.43603(19) & 0.78786(8) & 0.0221(4) \\ \text { O33 } & 0.42506(16) & -0.2921(2) & 0.57331(10) & 0.0308(5) \\ \text { O34 } & 0.59193(17) & -0.1572(2) & 0.54595(9) & 0.0282(4) \\ \text { O36 } & 0.59191(19) & -0.2314(2) & 0.65057(9) & 0.0308(4) \\ \text { S27 } & 0.84252(5) & 0.52334(6) & 0.73995(3) & 0.01897(15) \\ \text { S32 } & 0.55448(5) & -0.26334(6) & 0.58663(3) & 0.01882(15) \\ \text { H22A } & -0.168(3) & 1.102(3) & 0.3710(14) & 0.017(7)^{*} \\ \text { H25B } & 0.902(3) & 0.162(3) & 0.7333(14) & 0.019(7)^{*} \\ \text { H22B } & -0.266(3) & 1.177(3) & 0.3897(13) & 0.019(7)^{*} \\ \text { H25A } & 0.806(3) & 0.244(4) & 0.7016(17) & 0.039(10)^{*} \\ \text { H26B } & 0.787(3) & -0.150(4) & 0.7025(17) & 0.045(10)^{*} \\ \text { H15A } & -0.354(3) & 1.132(4) & 0.4725(17) & 0.043(10)^{*} \\ \text { H26A } & 0.905(4) & -0.078(4) & 0.7167(18) & 0.051(11)^{*} \\ \text { H15B } & -0.323(3) & 0.994(4) & 0.5063(18) & 0.043(10)^{*} \\ \text { H23 } & 0.627(4) & -0.042(5) & 0.6577(19) & 0.062(12)^{*} \\ & & & & \end{array}$

Atomic displacement parameters $\left(\AA^{2}\right)$

\begin{tabular}{lllllll}
\hline & $U^{11}$ & $U^{22}$ & $U^{33}$ & $U^{12}$ & $U^{13}$ & $U^{23}$ \\
\hline C1 & $0.0136(11)$ & $0.0182(13)$ & $0.0341(14)$ & $0.0017(9)$ & $-0.0030(10)$ & $-0.0012(10)$ \\
C2 & $0.0163(12)$ & $0.0241(14)$ & $0.0307(14)$ & $-0.0010(10)$ & $0.0023(10)$ & $0.0018(11)$ \\
C3 & $0.0134(11)$ & $0.0253(14)$ & $0.0324(14)$ & $0.0000(10)$ & $0.0060(10)$ & $-0.0028(11)$ \\
C4 & $0.0129(11)$ & $0.0224(13)$ & $0.0319(14)$ & $-0.0016(10)$ & $0.0021(10)$ & $-0.0035(11)$ \\
C5 & $0.0141(11)$ & $0.0245(14)$ & $0.0306(13)$ & $-0.0018(10)$ & $0.0047(10)$ & $-0.0030(11)$ \\
C6 & $0.0126(11)$ & $0.0194(13)$ & $0.0359(14)$ & $-0.0009(9)$ & $0.0002(10)$ & $-0.0011(11)$ \\
C7 & $0.0184(12)$ & $0.0250(14)$ & $0.0271(13)$ & $-0.0014(10)$ & $0.0005(10)$ & $0.0011(10)$ \\
C8 & $0.0160(12)$ & $0.0217(13)$ & $0.0301(13)$ & $0.0023(10)$ & $0.0008(10)$ & $-0.0031(10)$ \\
C9 & $0.0156(11)$ & $0.0249(14)$ & $0.0256(13)$ & $-0.0039(10)$ & $0.0015(10)$ & $-0.0012(10)$ \\
C10 & $0.0177(12)$ & $0.0224(14)$ & $0.0277(13)$ & $-0.0004(10)$ & $0.0051(10)$ & $-0.0012(10)$ \\
C12 & $0.0138(11)$ & $0.0196(13)$ & $0.0286(13)$ & $-0.0014(9)$ & $0.0050(10)$ & $-0.0004(10)$ \\
C14 & $0.0114(11)$ & $0.0187(13)$ & $0.0303(13)$ & $-0.0028(9)$ & $0.0000(9)$ & $-0.0012(10)$ \\
C16 & $0.0126(11)$ & $0.0201(13)$ & $0.0293(13)$ & $-0.0013(9)$ & $0.0033(9)$ & $0.0019(10)$ \\
C17 & $0.0170(12)$ & $0.0255(14)$ & $0.0244(12)$ & $-0.0037(10)$ & $0.0018(10)$ & $0.0002(10)$ \\
C18 & $0.0182(12)$ & $0.0219(13)$ & $0.0259(13)$ & $-0.0015(10)$ & $0.0000(10)$ & $-0.0001(10)$ \\
C19 & $0.0144(11)$ & $0.0218(13)$ & $0.0290(13)$ & $-0.0016(10)$ & $0.0029(10)$ & $0.0020(10)$ \\
C20 & $0.0166(12)$ & $0.0250(14)$ & $0.0290(13)$ & $0.0003(10)$ & $0.0010(10)$ & $0.0008(11)$ \\
C21 & $0.0209(12)$ & $0.0217(13)$ & $0.0270(13)$ & $-0.0021(10)$ & $0.0069(10)$ & $0.0000(10)$ \\
C24 & $0.0142(11)$ & $0.0256(14)$ & $0.0247(12)$ & $0.0026(10)$ & $0.0057(9)$ & $0.0020(10)$ \\
C30 & $0.0151(12)$ & $0.0286(15)$ & $0.0301(14)$ & $0.0051(10)$ & $0.0035(10)$ & $0.0020(11)$
\end{tabular}


supporting information

\begin{tabular}{lllllll}
\hline C35 & $0.0194(12)$ & $0.0225(14)$ & $0.0359(15)$ & $0.0023(10)$ & $0.0004(11)$ & $0.0014(11)$ \\
$\mathrm{N} 15$ & $0.0169(10)$ & $0.0226(12)$ & $0.0324(12)$ & $0.0037(9)$ & $0.0054(9)$ & $0.0062(10)$ \\
$\mathrm{N} 22$ & $0.0149(10)$ & $0.0217(12)$ & $0.0280(12)$ & $0.0019(9)$ & $0.0037(9)$ & $0.0004(9)$ \\
$\mathrm{N} 23$ & $0.0137(10)$ & $0.0245(12)$ & $0.0334(12)$ & $0.0027(9)$ & $-0.0012(9)$ & $-0.0001(10)$ \\
$\mathrm{N} 25$ & $0.0160(11)$ & $0.0187(12)$ & $0.0378(13)$ & $0.0028(9)$ & $0.0037(10)$ & $0.0035(10)$ \\
$\mathrm{N} 26$ & $0.0146(11)$ & $0.0202(12)$ & $0.0409(14)$ & $0.0014(9)$ & $0.0034(9)$ & $0.0005(10)$ \\
O11 & $0.0142(8)$ & $0.0238(10)$ & $0.0267(9)$ & $0.0021(7)$ & $0.0002(7)$ & $-0.0028(7)$ \\
O13 & $0.0184(9)$ & $0.0270(10)$ & $0.0310(10)$ & $0.0029(7)$ & $0.0019(7)$ & $-0.0023(8)$ \\
O28 & $0.0234(9)$ & $0.0202(9)$ & $0.0264(9)$ & $0.0013(7)$ & $0.0021(7)$ & $-0.0010(7)$ \\
O29 & $0.0202(9)$ & $0.0200(10)$ & $0.0358(10)$ & $-0.0005(7)$ & $0.0059(8)$ & $0.0036(8)$ \\
O31 & $0.0136(8)$ & $0.0236(10)$ & $0.0288(9)$ & $0.0045(7)$ & $0.0012(7)$ & $0.0060(7)$ \\
O33 & $0.0126(9)$ & $0.0248(10)$ & $0.0547(13)$ & $0.0006(7)$ & $0.0027(8)$ & $0.0066(9)$ \\
O34 & $0.0288(10)$ & $0.0214(10)$ & $0.0358(11)$ & $0.0005(8)$ & $0.0102(8)$ & $0.0067(8)$ \\
O36 & $0.0398(11)$ & $0.0225(10)$ & $0.0282(10)$ & $0.0011(8)$ & $-0.0038(8)$ & $0.0013(8)$ \\
S27 & $0.0124(3)$ & $0.0184(3)$ & $0.0264(3)$ & $0.0017(2)$ & $0.0034(2)$ & $0.0019(2)$ \\
S32 & $0.0117(3)$ & $0.0167(3)$ & $0.0281(3)$ & $0.0007(2)$ & $0.0021(2)$ & $0.0025(2)$ \\
& & & & & \\
\hline
\end{tabular}

Geometric parameters $\left(\stackrel{A}{A}{ }^{\circ}\right)$

\begin{tabular}{llll}
\hline $\mathrm{C} 1-\mathrm{O} 11$ & $1.391(3)$ & $\mathrm{C} 35-\mathrm{S} 32$ & $1.762(3)$ \\
$\mathrm{C} 1-\mathrm{C} 2$ & $1.401(4)$ & $\mathrm{C} 35-\mathrm{H} 35 \mathrm{C}$ & 0.98 \\
$\mathrm{C} 1-\mathrm{C} 10$ & $1.373(4)$ & $\mathrm{C} 35-\mathrm{H} 35 \mathrm{~B}$ & 0.98 \\
$\mathrm{C} 10-\mathrm{H} 10$ & 0.95 & $\mathrm{C} 35-\mathrm{H} 35 \mathrm{~A}$ & 0.98 \\
$\mathrm{C} 12-\mathrm{O} 13$ & $1.204(3)$ & $\mathrm{C} 4-\mathrm{C} 9$ & $1.430(4)$ \\
$\mathrm{C} 12-\mathrm{O} 11$ & $1.377(3)$ & $\mathrm{C} 4-\mathrm{C} 5$ & $1.407(4)$ \\
$\mathrm{C} 12-\mathrm{C} 16$ & $1.481(3)$ & $\mathrm{C} 5-\mathrm{H} 5$ & 0.95 \\
$\mathrm{C} 14-\mathrm{N} 22$ & $1.311(3)$ & $\mathrm{C} 5-\mathrm{C} 6$ & $1.396(4)$ \\
$\mathrm{C} 14-\mathrm{N} 15$ & $1.319(3)$ & $\mathrm{C} 6-\mathrm{C} 7$ & $1.413(4)$ \\
$\mathrm{C} 16-\mathrm{C} 21$ & $1.393(4)$ & $\mathrm{C} 6-\mathrm{C} 14$ & $1.486(3)$ \\
$\mathrm{C} 16-\mathrm{C} 17$ & $1.393(4)$ & $\mathrm{C} 7-\mathrm{H} 7$ & 0.95 \\
$\mathrm{C} 17-\mathrm{H} 17$ & 0.95 & $\mathrm{C} 7-\mathrm{C} 8$ & $1.369(4)$ \\
$\mathrm{C} 17-\mathrm{C} 18$ & $1.402(4)$ & $\mathrm{C} 8-\mathrm{H} 8$ & 0.95 \\
$\mathrm{C} 18-\mathrm{H} 18$ & 0.95 & $\mathrm{C} 8-\mathrm{C} 9$ & $1.412(4)$ \\
$\mathrm{C} 18-\mathrm{C} 19$ & $1.390(4)$ & $\mathrm{C} 9-\mathrm{C} 10$ & $1.403(4)$ \\
$\mathrm{C} 19-\mathrm{N} 23$ & $1.417(3)$ & $\mathrm{N} 15-\mathrm{H} 15 \mathrm{~B}$ & $0.85(4)$ \\
$\mathrm{C} 19-\mathrm{C} 20$ & $1.397(4)$ & $\mathrm{N} 15-\mathrm{H} 15 \mathrm{~A}$ & $0.93(4)$ \\
$\mathrm{C} 2-\mathrm{H} 2$ & 0.95 & $\mathrm{~N} 22-\mathrm{H} 22 \mathrm{~B}$ & $0.83(3)$ \\
$\mathrm{C} 2-\mathrm{C} 3$ & $1.379(4)$ & $\mathrm{N} 22-\mathrm{H} 22 \mathrm{~A}$ & $0.83(3)$ \\
$\mathrm{C} 20-\mathrm{H} 20$ & 0.95 & $\mathrm{~N} 23-\mathrm{H} 23$ & $0.99(4)$ \\
$\mathrm{C} 20-\mathrm{C} 21$ & $1.384(4)$ & $\mathrm{N} 25-\mathrm{H} 25 \mathrm{~B}$ & $0.83(3)$ \\
$\mathrm{C} 21-\mathrm{H} 21$ & 0.95 & $\mathrm{~N} 25-\mathrm{H} 25 \mathrm{~A}$ & $0.87(4)$ \\
$\mathrm{C} 24-\mathrm{N} 26$ & $1.325(3)$ & $\mathrm{N} 26-\mathrm{H} 26 \mathrm{~B}$ & $0.89(4)$ \\
$\mathrm{C} 24-\mathrm{N} 25$ & $1.302(4)$ & $\mathrm{N} 26-\mathrm{H} 26 \mathrm{~A}$ & $0.85(4)$ \\
$\mathrm{C} 24-\mathrm{N} 23$ & $1.357(3)$ & $\mathrm{O} 28-\mathrm{S} 27$ & $1.4515(19)$ \\
$\mathrm{C} 3-\mathrm{H} 3$ & 0.95 & $\mathrm{O} 29-\mathrm{S} 27$ & $1.4570(19)$ \\
$\mathrm{C} 3-\mathrm{C} 4$ & $1.398(4)$ & $\mathrm{O} 31-\mathrm{S} 27$ & $1.4700(18)$ \\
$\mathrm{C} 30-\mathrm{S} 27$ & $1.764(3)$ & $\mathrm{O} 34-\mathrm{S} 32$ & $1.4545(18)$ \\
$\mathrm{C} 30-\mathrm{H} 30 \mathrm{C}$ & 0.98 & $(19)$
\end{tabular}




\begin{tabular}{|c|c|c|c|}
\hline $\mathrm{C} 30-\mathrm{H} 30 \mathrm{~B}$ & 0.98 & $\mathrm{O} 36-\mathrm{S} 32$ & $1.448(2)$ \\
\hline $\mathrm{C} 30-\mathrm{H} 30 \mathrm{~A}$ & 0.98 & & \\
\hline $\mathrm{C} 10-\mathrm{C} 1-\mathrm{O} 11$ & $116.5(2)$ & $\mathrm{C} 21-\mathrm{C} 20-\mathrm{H} 20$ & 120.2 \\
\hline $\mathrm{C} 10-\mathrm{C} 1-\mathrm{C} 2$ & $122.3(2)$ & $\mathrm{C} 19-\mathrm{C} 20-\mathrm{H} 20$ & 120.2 \\
\hline $\mathrm{O} 11-\mathrm{C} 1-\mathrm{C} 2$ & $121.2(2)$ & $\mathrm{C} 20-\mathrm{C} 21-\mathrm{C} 16$ & $120.7(3)$ \\
\hline $\mathrm{C} 3-\mathrm{C} 2-\mathrm{C} 1$ & $118.7(2)$ & $\mathrm{C} 20-\mathrm{C} 21-\mathrm{H} 21$ & 119.6 \\
\hline $\mathrm{C} 3-\mathrm{C} 2-\mathrm{H} 2$ & 120.6 & $\mathrm{C} 16-\mathrm{C} 21-\mathrm{H} 21$ & 119.6 \\
\hline $\mathrm{C} 1-\mathrm{C} 2-\mathrm{H} 2$ & 120.6 & $\mathrm{~N} 25-\mathrm{C} 24-\mathrm{N} 26$ & $121.0(2)$ \\
\hline $\mathrm{C} 2-\mathrm{C} 3-\mathrm{C} 4$ & $120.8(2)$ & $\mathrm{N} 25-\mathrm{C} 24-\mathrm{N} 23$ & $123.3(2)$ \\
\hline $\mathrm{C} 2-\mathrm{C} 3-\mathrm{H} 3$ & 119.6 & $\mathrm{~N} 26-\mathrm{C} 24-\mathrm{N} 23$ & $115.7(2)$ \\
\hline $\mathrm{C} 4-\mathrm{C} 3-\mathrm{H} 3$ & 119.6 & $\mathrm{~S} 27-\mathrm{C} 30-\mathrm{H} 30 \mathrm{~A}$ & 109.5 \\
\hline $\mathrm{C} 3-\mathrm{C} 4-\mathrm{C} 5$ & $121.2(2)$ & $\mathrm{S} 27-\mathrm{C} 30-\mathrm{H} 30 \mathrm{~B}$ & 109.5 \\
\hline $\mathrm{C} 3-\mathrm{C} 4-\mathrm{C} 9$ & $119.7(2)$ & $\mathrm{H} 30 \mathrm{~A}-\mathrm{C} 30-\mathrm{H} 30 \mathrm{~B}$ & 109.5 \\
\hline $\mathrm{C} 5-\mathrm{C} 4-\mathrm{C} 9$ & $119.1(2)$ & $\mathrm{S} 27-\mathrm{C} 30-\mathrm{H} 30 \mathrm{C}$ & 109.5 \\
\hline $\mathrm{C} 6-\mathrm{C} 5-\mathrm{C} 4$ & $121.5(2)$ & $\mathrm{H} 30 \mathrm{~A}-\mathrm{C} 30-\mathrm{H} 30 \mathrm{C}$ & 109.5 \\
\hline $\mathrm{C} 6-\mathrm{C} 5-\mathrm{H} 5$ & 119.3 & $\mathrm{H} 30 \mathrm{~B}-\mathrm{C} 30-\mathrm{H} 30 \mathrm{C}$ & 109.5 \\
\hline $\mathrm{C} 4-\mathrm{C} 5-\mathrm{H} 5$ & 119.3 & $\mathrm{~S} 32-\mathrm{C} 35-\mathrm{H} 35 \mathrm{~A}$ & 109.5 \\
\hline $\mathrm{C} 5-\mathrm{C} 6-\mathrm{C} 7$ & $119.0(2)$ & $\mathrm{S} 32-\mathrm{C} 35-\mathrm{H} 35 \mathrm{~B}$ & 109.5 \\
\hline $\mathrm{C} 5-\mathrm{C} 6-\mathrm{C} 14$ & $119.6(2)$ & $\mathrm{H} 35 \mathrm{~A}-\mathrm{C} 35-\mathrm{H} 35 \mathrm{~B}$ & 109.5 \\
\hline $\mathrm{C} 7-\mathrm{C} 6-\mathrm{C} 14$ & $121.3(2)$ & $\mathrm{S} 32-\mathrm{C} 35-\mathrm{H} 35 \mathrm{C}$ & 109.5 \\
\hline $\mathrm{C} 8-\mathrm{C} 7-\mathrm{C} 6$ & $120.1(2)$ & $\mathrm{H} 35 \mathrm{~A}-\mathrm{C} 35-\mathrm{H} 35 \mathrm{C}$ & 109.5 \\
\hline $\mathrm{C} 8-\mathrm{C} 7-\mathrm{H} 7$ & 120 & $\mathrm{H} 35 \mathrm{~B}-\mathrm{C} 35-\mathrm{H} 35 \mathrm{C}$ & 109.5 \\
\hline $\mathrm{C} 6-\mathrm{C} 7-\mathrm{H} 7$ & 120 & $\mathrm{C} 14-\mathrm{N} 15-\mathrm{H} 15 \mathrm{~A}$ & $116(2)$ \\
\hline $\mathrm{C} 7-\mathrm{C} 8-\mathrm{C} 9$ & $122.3(2)$ & $\mathrm{C} 14-\mathrm{N} 15-\mathrm{H} 15 \mathrm{~B}$ & $120(3)$ \\
\hline $\mathrm{C} 7-\mathrm{C} 8-\mathrm{H} 8$ & 118.8 & $\mathrm{H} 15 \mathrm{~A}-\mathrm{N} 15-\mathrm{H} 15 \mathrm{~B}$ & $121(3)$ \\
\hline $\mathrm{C} 9-\mathrm{C} 8-\mathrm{H} 8$ & 118.8 & $\mathrm{C} 14-\mathrm{N} 22-\mathrm{H} 22 \mathrm{~A}$ & $123(2)$ \\
\hline $\mathrm{C} 10-\mathrm{C} 9-\mathrm{C} 8$ & $123.3(2)$ & $\mathrm{C} 14-\mathrm{N} 22-\mathrm{H} 22 \mathrm{~B}$ & $121(2)$ \\
\hline $\mathrm{C} 10-\mathrm{C} 9-\mathrm{C} 4$ & $118.7(2)$ & $\mathrm{H} 22 \mathrm{~A}-\mathrm{N} 22-\mathrm{H} 22 \mathrm{~B}$ & $116(3)$ \\
\hline $\mathrm{C} 8-\mathrm{C} 9-\mathrm{C} 4$ & $117.9(2)$ & $\mathrm{C} 24-\mathrm{N} 23-\mathrm{C} 19$ & $127.7(2)$ \\
\hline $\mathrm{C} 1-\mathrm{C} 10-\mathrm{C} 9$ & $119.6(2)$ & $\mathrm{C} 24-\mathrm{N} 23-\mathrm{H} 23$ & $115(2)$ \\
\hline $\mathrm{C} 1-\mathrm{C} 10-\mathrm{H} 10$ & 120.2 & $\mathrm{C} 19-\mathrm{N} 23-\mathrm{H} 23$ & $117(2)$ \\
\hline $\mathrm{C} 9-\mathrm{C} 10-\mathrm{H} 10$ & 120.2 & $\mathrm{C} 24-\mathrm{N} 25-\mathrm{H} 25 \mathrm{~B}$ & $121(2)$ \\
\hline $\mathrm{O} 13-\mathrm{C} 12-\mathrm{O} 11$ & $122.9(2)$ & $\mathrm{C} 24-\mathrm{N} 25-\mathrm{H} 25 \mathrm{~A}$ & $122(2)$ \\
\hline $\mathrm{O} 13-\mathrm{C} 12-\mathrm{C} 16$ & $125.5(2)$ & $\mathrm{H} 25 \mathrm{~B}-\mathrm{N} 25-\mathrm{H} 25 \mathrm{~A}$ & $116(3)$ \\
\hline $\mathrm{O} 11-\mathrm{C} 12-\mathrm{C} 16$ & $111.6(2)$ & $\mathrm{C} 24-\mathrm{N} 26-\mathrm{H} 26 \mathrm{~B}$ & $122(2)$ \\
\hline $\mathrm{N} 22-\mathrm{C} 14-\mathrm{N} 15$ & $119.1(2)$ & $\mathrm{C} 24-\mathrm{N} 26-\mathrm{H} 26 \mathrm{~A}$ & $120(3)$ \\
\hline $\mathrm{N} 22-\mathrm{C} 14-\mathrm{C} 6$ & $121.9(2)$ & $\mathrm{H} 26 \mathrm{~B}-\mathrm{N} 26-\mathrm{H} 26 \mathrm{~A}$ & $117(4)$ \\
\hline $\mathrm{N} 15-\mathrm{C} 14-\mathrm{C} 6$ & $119.0(2)$ & $\mathrm{C} 12-\mathrm{O} 11-\mathrm{C} 1$ & $118.4(2)$ \\
\hline $\mathrm{C} 17-\mathrm{C} 16-\mathrm{C} 21$ & $119.3(2)$ & $\mathrm{O} 28-\mathrm{S} 27-\mathrm{O} 29$ & $113.51(11)$ \\
\hline $\mathrm{C} 17-\mathrm{C} 16-\mathrm{C} 12$ & $118.1(2)$ & $\mathrm{O} 28-\mathrm{S} 27-\mathrm{O} 31$ & $112.04(11)$ \\
\hline $\mathrm{C} 21-\mathrm{C} 16-\mathrm{C} 12$ & $122.6(2)$ & $\mathrm{O} 29-\mathrm{S} 27-\mathrm{O} 31$ & $111.41(11)$ \\
\hline $\mathrm{C} 16-\mathrm{C} 17-\mathrm{C} 18$ & $120.7(2)$ & $\mathrm{O} 28-\mathrm{S} 27-\mathrm{C} 30$ & $106.72(12)$ \\
\hline $\mathrm{C} 16-\mathrm{C} 17-\mathrm{H} 17$ & 119.7 & $\mathrm{O} 29-\mathrm{S} 27-\mathrm{C} 30$ & $106.21(12)$ \\
\hline $\mathrm{C} 18-\mathrm{C} 17-\mathrm{H} 17$ & 119.7 & $\mathrm{O} 31-\mathrm{S} 27-\mathrm{C} 30$ & $106.39(12)$ \\
\hline $\mathrm{C} 19-\mathrm{C} 18-\mathrm{C} 17$ & $119.0(2)$ & $\mathrm{O} 36-\mathrm{S} 32-\mathrm{O} 33$ & $113.48(13)$ \\
\hline $\mathrm{C} 19-\mathrm{C} 18-\mathrm{H} 18$ & 120.5 & $\mathrm{O} 36-\mathrm{S} 32-\mathrm{O} 34$ & $111.82(12)$ \\
\hline $\mathrm{C} 17-\mathrm{C} 18-\mathrm{H} 18$ & 120.5 & $\mathrm{O} 33-\mathrm{S} 32-\mathrm{O} 34$ & $110.96(12)$ \\
\hline
\end{tabular}




$\begin{array}{llll}\mathrm{C} 18-\mathrm{C} 19-\mathrm{C} 20 & 120.6(2) & \mathrm{O} 36-\mathrm{S} 32-\mathrm{C} 35 & 106.84(13) \\ \mathrm{C} 18-\mathrm{C} 19-\mathrm{N} 23 & 122.1(2) & \mathrm{O} 33-\mathrm{S} 32-\mathrm{C} 35 & 105.73(12) \\ \mathrm{C} 20-\mathrm{C} 19-\mathrm{N} 23 & 117.2(2) & \mathrm{O} 34-\mathrm{S} 32-\mathrm{C} 35 & 107.57(12) \\ \mathrm{C} 21-\mathrm{C} 20-\mathrm{C} 19 & 119.7(3) & & \end{array}$

Hydrogen-bond geometry $\left(A,{ }^{\circ}\right)$

$\operatorname{Cg}(C)$ is the center of gravity of phenyl ring $C$.

\begin{tabular}{|c|c|c|c|c|}
\hline$D-\mathrm{H} \cdots A$ & $D-\mathrm{H}$ & $\mathrm{H} \cdots A$ & $D \cdots A$ & $D-\mathrm{H} \cdots A$ \\
\hline $\mathrm{C} 18-\mathrm{H} 18 \cdots \mathrm{O} 13^{\mathrm{i}}$ & 0.95 & 2.64 & $3.419(3)$ & 140 \\
\hline $\mathrm{C} 30-\mathrm{H} 30 A \cdots \mathrm{O} 36^{\mathrm{ii}}$ & 0.98 & 2.36 & $3.314(3)$ & 165 \\
\hline $\mathrm{N} 15-\mathrm{H} 15 A \cdots \mathrm{O} 33^{\mathrm{iii}}$ & $0.93(4)$ & $1.97(4)$ & $2.854(3)$ & $159(3)$ \\
\hline $\mathrm{N} 15-\mathrm{H} 15 A \cdots \mathrm{O} 34^{\mathrm{iii}}$ & $0.93(4)$ & $2.63(4)$ & $3.327(3)$ & $132(3)$ \\
\hline $\mathrm{N} 15-\mathrm{H} 15 A \cdots \mathrm{S} 32^{\mathrm{iii}}$ & $0.93(4)$ & $2.75(4)$ & $3.612(2)$ & $154(3)$ \\
\hline $\mathrm{N} 15-\mathrm{H} 15 B \cdots \mathrm{O} 34^{\mathrm{iv}}$ & $0.85(4)$ & $2.00(4)$ & $2.830(3)$ & $164(4)$ \\
\hline $\mathrm{N} 22-\mathrm{H} 22 A \cdots \mathrm{O} 31^{\mathrm{v}}$ & $0.83(3)$ & $2.12(3)$ & $2.928(3)$ & $162(3)$ \\
\hline $\mathrm{N} 22-\mathrm{H} 22 B \cdots \mathrm{O} 33^{\mathrm{iii}}$ & $0.83(3)$ & $2.31(3)$ & $3.018(3)$ & $144(3)$ \\
\hline $\mathrm{N} 23-\mathrm{H} 23 \cdots \mathrm{O} 36$ & $0.99(4)$ & $1.88(5)$ & $2.836(3)$ & $163(4)$ \\
\hline $\mathrm{N} 23-\mathrm{H} 23 \cdots \mathrm{S} 32$ & $0.99(4)$ & $2.72(4)$ & $3.683(2)$ & $166(3)$ \\
\hline $\mathrm{N} 25-\mathrm{H} 25 A \cdots \mathrm{O} 28$ & $0.87(4)$ & $2.00(4)$ & $2.827(3)$ & $159(3)$ \\
\hline $\mathrm{N} 25-\mathrm{H} 25 A \cdots \mathrm{S} 27$ & $0.87(4)$ & $2.86(4)$ & $3.558(2)$ & $139(3)$ \\
\hline $\mathrm{N} 25-\mathrm{H} 25 B \cdots \mathrm{O} 29^{\mathrm{vi}}$ & $0.83(3)$ & $2.12(3)$ & $2.931(3)$ & $167(3)$ \\
\hline $\mathrm{N} 26-\mathrm{H} 26 A \cdots \mathrm{O} 31^{\mathrm{vi}}$ & $0.85(4)$ & $2.10(4)$ & $2.916(3)$ & $163(4)$ \\
\hline $\mathrm{N} 26-\mathrm{H} 26 A \cdots \mathrm{S} 27^{\mathrm{vi}}$ & $0.85(4)$ & $3.01(4)$ & $3.799(2)$ & $156(3)$ \\
\hline $\mathrm{N} 26-\mathrm{H} 26 B \cdots \mathrm{O} 29^{\text {vii }}$ & $0.89(4)$ & $2.46(4)$ & $2.925(3)$ & $113(3)$ \\
\hline $\mathrm{N} 26-\mathrm{H} 26 B \cdots \mathrm{O} 36$ & $0.89(4)$ & $2.45(4)$ & $3.174(3)$ & $139(3)$ \\
\hline $\mathrm{C} 30-\mathrm{H} 30 B^{\cdots} \cdot C g(\mathrm{C})^{\mathrm{vii}}$ & 0.98 & 2.96 & $3.405(3)$ & 109 \\
\hline
\end{tabular}

Symmetry codes: (i) $-x+1, y-1 / 2,-z+3 / 2$; (ii) $x, y+1, z$; (iii) $-x,-y+1,-z+1$; (iv) $x-1, y+1, z$; (v) $x-1,-y+3 / 2, z-1 / 2$; (vi) $-x+2, y-1 / 2,-z+3 / 2$; (vii) $x$, $y-1, z$. 